\title{
Reliability of Interconnection Networks Modeled by Cartesian Product Digraphs
}

\author{
Chao Yang and Jun-Ming Xu \\ Department of Mathematics, University of Science and Technology of China, Hefei 230026, China
}

\begin{abstract}
We determine that the connectivity and the edgeconnectivity of the Cartesian product $G_{1} \times G_{2}$ of two strongly connected and finite digraphs $G_{1}$ and $G_{2}$ are equal to $\min \left\{n_{1} \kappa_{2}, n_{2} \kappa_{1}, \delta_{1}^{+}+\delta_{2}^{+}, \delta_{1}^{-}+\delta_{2}^{-}\right\}$and $\min \left\{n_{1} \lambda_{2}, n_{2} \lambda_{1}, \delta_{1}^{+}+\delta_{2}^{+}, \delta_{1}^{-}+\delta_{2}^{-}\right\}$, respectively, where $n_{i}$, $\kappa_{i}, \lambda_{i}, \delta_{i}^{+}, \delta_{i}^{-}$are the order, the connectivity, the edgeconnectivity, the minimum out-degree and the minimum in-degree of $G_{i}$, respectively, for $i=1,2$. (C) 2008 Wiley Periodicals, Inc. NETWORKS, Vol. 52(4), 202-205 2008
\end{abstract}

Keywords: connectivity; edge-connectivity; Cartesian product digraphs

\section{INTRODUCTION}

Graph theory has become one of the most powerful mathematical tools in the analysis and study of the architecture of an interconnection network. It is well known that when the underlying topology of an interconnection network is modeled by a connected graph $G=(V, E)$, where $V$ is the set of processors and $E$ is the set of communication links in the network, the connectivity $\kappa(G)$ and the edge-connectivity $\lambda(G)$ are two important features determining reliability and fault tolerance of the network. It is also well known that, for designing large-scale interconnection networks, the Cartesian product is an important method to obtain large graphs from smaller ones, with a number of parameters that can be easily calculated from the corresponding parameters for those small initial graphs. The Cartesian product preserves many nice properties such as regularity, existence of Hamilton cycles and Euler circuits, and transitivity of the initial graphs (see, e.g., [10]). In this note, we deal with the connectivity and the edge-connectivity of the Cartesian product of graphs.

We use the symbols $n_{i}, \delta_{i}, \kappa_{i}$, and $\lambda_{i}$ to denote the order, the minimum degree, the connectivity, and the edge-connectivity

Received December 2006; accepted September 2007

Correspondence to: J.-M. Xu; e-mail: xujm@ustc.edu.cn

Contract grant sponsor: NNSF of China; Contract grant number: 10671191 DOI 10.1002/net.20231

Published online 11 January 2008 in Wiley InterScience (www. interscience.wiley.com).

C) 2008 Wiley Periodicals, Inc. of a graph $G_{i}$, respectively, for $i=1,2$. In 1957, Sabidussi [8] first discussed the connectivity of the Cartesian product $G_{1} \times G_{2}$ of two undirected graphs $G_{1}$ and $G_{2}$ and proved that $\kappa\left(G_{1} \times G_{2}\right) \geq \kappa_{1}+\kappa_{2}$ if $G_{1}$ and $G_{2}$ are connected. In 1998, Xu [9] generalized this result to strongly connected digraphs. In 1999, Chiue and Shieh [4] proved that for connected undirected graphs $G_{1}$ and $G_{2}, \lambda\left(G_{1} \times G_{2}\right) \geq \lambda_{1}+\lambda_{2}$. The authors $[12,13]$ completely determined the connectivity and the edge-connectivity of the Cartesian product of two connected undirected graphs $G_{1}$ and $G_{2}$ : namely,

$$
\begin{aligned}
& \kappa\left(G_{1} \times G_{2}\right)=\min \left\{n_{1} \kappa_{2}, n_{2} \kappa_{1}, \delta_{1}+\delta_{2}\right\}, \\
& \lambda\left(G_{1} \times G_{2}\right)=\min \left\{n_{1} \lambda_{2}, n_{2} \lambda_{1}, \delta_{1}+\delta_{2}\right\} .
\end{aligned}
$$

Very recently, Lu et al. [6] have further considered the super edge-connectivity of Cartesian product graphs.

Balbuena et al. [1] considered a generalization of the Cartesian product of graphs, the product graph $G_{1} \times G_{2}$ of two undirected graphs $G_{1}$ and $G_{2}$, and obtained

$$
\kappa\left(G_{1} \times G_{2}\right) \geq \min \left\{\kappa_{1} n_{2},\left(\delta_{1}+1\right) \kappa_{2}, \delta_{1}+\delta_{2}\right\} .
$$

Moreover, they stated some sufficient conditions that guarantee these product graphs to be maximally connected or superconnected.

Motivated by the technique in [1], in this note, we completely determine the connectivity and the edge-connectivity of the Cartesian product of two digraphs $G_{1}$ and $G_{2}$ as follows:

$$
\begin{aligned}
& \kappa\left(G_{1} \times G_{2}\right)=\min \left\{n_{1} \kappa_{2}, n_{2} \kappa_{1}, \delta_{1}^{+}+\delta_{2}^{+}, \delta_{1}^{-}+\delta_{2}^{-}\right\}, \\
& \lambda\left(G_{1} \times G_{2}\right)=\min \left\{n_{1} \lambda_{2}, n_{2} \lambda_{1}, \delta_{1}^{+}+\delta_{2}^{+}, \delta_{1}^{-}+\delta_{2}^{-}\right\},
\end{aligned}
$$

where $G_{i}$ is a strongly connected digraph with minimum outdegree $\delta_{i}^{+}$and minimum in-degree $\delta_{i}^{-}$, for $i=1,2$.

The proofs of these results are in Section 3. In Section 2 , we present some preliminaries and the definition of the Cartesian product of two digraphs.

\section{PRELIMINARIES}

We follow [11] for graph-theoretical terminology and notation not defined here. Let $G=(V, E)$ be a strongly connected digraph. A subset $S \subset V(G)$ (resp. $B \subseteq E(G)$ ) is said 
to be a separating set (an edge-separating set) of $G$ if $G-S$ (resp. $G-B$ ) is not strongly connected. The connectivity of $G$ is defined as $\kappa(G)=\min \{|S|: S$ is a separating set of $G\}$ if $G$ is not a complete digraph; and $\kappa(G)=n-1$ if $G$ is a complete digraph of order $n$. The edge-connectivity of $G$ is defined as $\lambda(G)=\min \{|B|: B$ is an edge-separating set of $G\}$. For any digraph $G$, we have $\kappa \leq \lambda \leq \delta=\min \left\{\delta^{+}, \delta^{-}\right\}$.

Let $D$ and $D^{\prime}$ be two disjoint nonempty proper subsets of $V(G)$ or subgraphs of $G$. The symbols $N_{G}^{+}(D)$ and $N_{G}^{-}(D)$ denote the set of out-neighbors of $D$ and the set of in-neighbors of $D, E_{G}^{+}(D)$, and $E_{G}^{-}(D)$ denote the set of out-going edges of $D$ and the set of in-coming edges of $D$, respectively, and $E_{G}\left(D, D^{\prime}\right)$ denotes the set of edges with tails in $D$ and heads in $D^{\prime}$. The following two facts are simple and useful for the proofs of our main results.

Remark 1. Let G be a digraph. If it is not strongly connected and has a finite number of strongly connected components, then $G$ has at least two strongly connected components $C$ and $C^{\prime}$ such that $N_{G}^{+}(C)=\emptyset$ and $N_{G}^{-}\left(C^{\prime}\right)=\emptyset$.

Remark 2. Let $G$ be a digraph and let $D$ be a nonempty proper subset of $V(G)$. Then $|D|+\left|N_{G}^{+}(D)\right| \geq \delta^{+}(G)+1$ and $|D|+\left|N_{G}^{-}(D)\right| \geq \delta^{-}(G)+1$. Also $|D|+\left|E_{G}^{+}(D)\right| \geq \delta^{+}(G)+1$ and $|D|+\left|E_{G}^{-}(D)\right| \geq \delta^{-}(G)+1$.

The Cartesian product digraph $G_{1} \times G_{2}=(V, E)$ of digraphs $G_{1}=\left(V_{1}, E_{1}\right)$ and $G_{2}=\left(V_{2}, E_{2}\right)$ has the vertex set $V=V_{1} \times V_{2}=\left\{x y: x \in V_{1}, y \in V_{2}\right\}$, and $\left(x_{1} x_{2}, y_{1} y_{2}\right) \in E$ if and only if either $x_{1}=y_{1}$ and $\left(x_{2}, y_{2}\right) \in E_{2}$ or $x_{2}=y_{2}$ and $\left(x_{1}, y_{1}\right) \in E_{1}$. Obviously, $\delta^{+}\left(G_{1} \times G_{2}\right)=\delta_{1}^{+}+\delta_{2}^{+}$and $\delta^{-}\left(G_{1} \times G_{2}\right)=\delta_{1}^{-}+\delta_{2}^{-}$.

We now introduce some notations for convenience. For $x \in V\left(G_{1}\right)$ and $y \in V\left(G_{2}\right)$, the symbols $G_{2}^{x}$ and $G_{1}^{y}$ denote the subgraph of $G_{1} \times G_{2}$ induced by $\{x\} \times V\left(G_{2}\right)$ and $V\left(G_{1}\right) \times\{y\}$, respectively. For a separating set $S$ and an edge-separating set $B$ of $G_{1} \times G_{2}$, denote

$$
\begin{gathered}
S_{x}=S \cap V\left(G_{2}^{x}\right), \quad S^{y}=S \cap V\left(G_{1}^{y}\right), \\
B_{x}=B \cap E\left(G_{2}^{x}\right), \quad B^{y}=B \cap E\left(G_{1}^{y}\right), \\
B_{x z}=B \cap E_{G}\left(G_{2}^{x}, G_{2}^{z}\right) \text { if }(x, z) \in E\left(G_{1}\right) .
\end{gathered}
$$

For a strongly connected component $C$ of $G-S($ or $G-B$ ), denote

$$
\begin{aligned}
& C_{1}=\left\{x \in V_{1}: x y \in V(C) \text { for some } y \in V_{2}\right\}, \\
& C_{2}=\left\{y \in V_{2}: x y \in V(C) \text { for some } x \in V_{1}\right\} .
\end{aligned}
$$

Lemma 1. Let $G_{1}$ and $G_{2}$ be two strongly connected digraphs, $S$ and $B$ be a separating set and an edge-separating set of $G_{1} \times G_{2}$, respectively, and $C$ be a strongly connected component of $G_{1} \times G_{2}-S$ (resp. $\left.G_{1} \times G_{2}-B\right)$ without out-neighbors. If $C_{1} \neq V_{1}$, then there exists an edge $\left(x, x^{\prime}\right) \in E_{G_{1}}^{+}\left(C_{1}\right)$ such that

$$
\left|S_{x}\right|+\left|S_{x^{\prime}}\right| \geq \delta_{2}^{+}+1 \quad\left(\text { resp. }\left|B_{x}\right|+\left|B_{x x^{\prime}}\right| \geq \delta_{2}^{+}+1\right) .
$$

Proof. Let $G=G_{1} \times G_{2}$. Since $C_{1} \neq V_{1}$ and $G_{1}$ is strongly connected, $E_{G_{1}}^{+}\left(C_{1}\right) \neq \varnothing$ and there exist $x \in C_{1}$ and $x^{\prime} \in V_{1} \backslash C_{1}$ such that $\left(x, x^{\prime}\right) \in E_{G_{1}}^{+}\left(C_{1}\right)$. Let $D=V(C) \cap$ $V\left(G_{2}^{x}\right)$ and $F=N_{G}^{+}(D) \cap V\left(G_{2}^{x^{\prime}}\right)$. Then

$N_{G_{2}^{x}}^{+}(D) \subseteq S_{x}, F \subseteq S_{x^{\prime}} \quad\left(\right.$ resp. $\left.E_{G_{2}^{x}}^{+}(D) \subseteq B_{x}, E_{G}(D, F) \subseteq B_{x x^{\prime}}\right)$

since $C$ has no out-neighbors in $G-S$ (resp. $G-B$ ), which means that

$$
\begin{array}{ll} 
& \left|S_{x}\right|+\left|S_{x^{\prime}}\right| \geq\left|N_{G_{2}^{x}}^{+}(D)\right|+|F| \\
\text { resp. } & \left.\left|B_{x}\right|+\left|B_{x x^{\prime}}\right| \geq\left|E_{G_{2}^{x}}^{+}(D)\right|+\left|E_{G}(D, F)\right|\right) .
\end{array}
$$

Note that $|F|=|D|=\left|E_{G}(D, F)\right|$ since $\left(x, x^{\prime}\right) \in E\left(G_{1}\right)$. It follows from (2) that

$$
\begin{array}{ll} 
& \left|S_{x}\right|+\left|S_{x^{\prime}}\right| \geq\left|N_{G_{2}^{x}}^{+}(D)\right|+|D| \\
\text { (resp. } & \left.\left|B_{x}\right|+\left|B_{x x^{\prime}}\right| \geq\left|E_{G_{2}^{x}}^{+}(D)\right|+|D|\right) .
\end{array}
$$

It follows from (3) and Lemma 2 that

$$
\left|S_{x}\right|+\left|S_{x^{\prime}}\right| \geq \delta_{2}^{+}+1 \quad\left(\text { resp. }\left|B_{x}\right|+\left|B_{x x^{\prime}}\right| \geq \delta_{2}^{+}+1\right)
$$

as required.

\section{MAIN RESULT}

Theorem 1. For every two nontrivial strongly connected digraphs $G_{1}$ and $G_{2}$,

$$
\kappa\left(G_{1} \times G_{2}\right)=\min \left\{n_{1} \kappa_{2}, n_{2} \kappa_{1}, \delta_{1}^{+}+\delta_{2}^{+}, \delta_{1}^{-}+\delta_{2}^{-}\right\} .
$$

Proof. Let $G=G_{1} \times G_{2}$. Clearly,

$$
\kappa(G) \leq \min \left\{\delta^{+}(G), \delta^{-}(G)\right\}=\min \left\{\delta_{1}^{+}+\delta_{2}^{+}, \delta_{1}^{-}+\delta_{2}^{-}\right\} .
$$

If $G_{2}$ is not a complete digraph, let $S_{0}$ be a minimum separating set of $G_{2}$. Then $V_{1} \times S_{0}$ is a separating set of $G$, which implies $\kappa(G) \leq n_{1} \kappa_{2}$. If $G_{2}$ is a complete digraph, then $\kappa_{2}=\delta_{2}^{+}$, therefore

$$
\kappa(G) \leq \delta_{1}^{+}+\delta_{2}^{+} \leq\left(\delta_{1}^{+}+1\right) \delta_{2}^{+} \leq n_{1} \kappa_{2} .
$$

By symmetry, we have $\kappa(G) \leq n_{2} \kappa_{1}$.

So it remains to show

$$
\kappa\left(G_{1} \times G_{2}\right) \geq \min \left\{\kappa_{1} n_{2}, \kappa_{2} n_{1}, \delta_{1}^{+}+\delta_{2}^{+}, \delta_{1}^{-}+\delta_{2}^{-}\right\} .
$$

Evidently, $G$ is not a complete digraph since neither $G_{1}$ nor $G_{2}$ is trivial. Let $S$ be a minimum separating set in $G$.

Assume $C_{1} \neq V_{1}$ and $C_{2} \neq V_{2}$ for each strongly connected component $C$ of $G-S$. Then

$$
\left|S_{x}\right| \geq 1 \text { for each } x \in V_{1} .
$$

Otherwise, there is a strongly connected component $C^{\prime}$ containing $G_{2}^{x}$ for some $x \in V_{1}$, which implies $C_{2}^{\prime}=V_{2}$, a contradiction. Let $C$ be a strongly connected component of $G-S$ without out-neighbors. Since $C_{1} \neq V_{1}$, by Lemma 1 
there exist $x \in C_{1}$ and $x^{\prime} \in V_{1} \backslash C_{1}$ such that $\left|S_{x}\right|+\left|S_{x^{\prime}}\right| \geq$ $\delta_{2}^{+}+1$. It follows from (4) that

$$
\begin{aligned}
|S| & =\left|S_{x}\right|+\left|S_{x^{\prime}}\right|+\sum_{z \in V_{1} \backslash\left\{x, x^{\prime}\right\}}\left|S_{z}\right| \\
& \geq \delta_{2}^{+}+1+\left(n_{1}-2\right) \\
& \geq \delta_{2}^{+}+1+\left(\delta_{1}^{+}+1\right)-2 \\
& =\delta_{1}^{+}+\delta_{2}^{+} .
\end{aligned}
$$

Now, suppose there exists such a strongly connected component $C$ of $G-S$ where either $C_{1}=V_{1}$ or $C_{2}=V_{2}$. By symmetry, we can without loss of generality assume $C_{1}=V_{1}$. By Lemma 1, let $C^{\prime}(\neq C)$ be a strongly connected component without out-neighbors in $G-S$ (if $C^{\prime}$ has no in-neighbors, the proof is similar). Then

$$
\left|S_{x}\right| \geq \kappa_{2} \quad \text { if } x \in C_{1}^{\prime} .
$$

If $C_{1}^{\prime}=V_{1}$, then from (5) we have

$$
|S| \geq \sum_{x \in C_{1}^{\prime}=V_{1}}\left|S_{x}\right| \geq n_{1} \kappa_{2} .
$$

If $C_{1}^{\prime} \neq V_{1}$, then by Lemma 1 there exist $x \in C_{1}^{\prime}$ and $x^{\prime} \in V_{1} \backslash C_{1}^{\prime}$ such that $\left|S_{x}\right|+\left|S_{x^{\prime}}\right| \geq \delta_{2}^{+}+1$. And we can also see that

$$
\left|S_{z}\right| \geq 1 \quad \text { for each } z \in N_{G_{1}}^{+}\left(C_{1}^{\prime}\right) .
$$

It follows from (1), (6), and Lemma 2 that

$$
\begin{aligned}
|S| & \geq\left|S_{x}\right|+\left|S_{x^{\prime}}\right|+\sum_{z \in C_{1}^{\prime} \backslash\{x\}}\left|S_{z}\right|+\sum_{z \in N_{G_{1}}^{+}\left(C_{1}^{\prime}\right) \backslash\left\{x^{\prime}\right\}}\left|S_{z}\right| \\
& \geq \delta_{2}^{+}+1+\left|C_{1}^{\prime}\right|-1+\left|N_{G_{1}}^{+}\left(C_{1}^{\prime}\right)\right|-1 \\
& =\delta_{2}^{+}+\left(\left|C_{1}^{\prime}\right|+\left|N_{G_{1}}^{+}\left(C_{1}^{\prime}\right)\right|\right)-1 \\
& \geq \delta_{2}^{+}+\left(\delta_{1}^{+}+1\right)-1 \\
& =\delta_{1}^{+}+\delta_{2}^{+} .
\end{aligned}
$$

This completes the proof.

Corollary 1. ([13]) For every two nontrivial connected undirected graphs $G_{1}$ and $G_{2}$,

$$
\kappa\left(G_{1} \times G_{2}\right)=\min \left\{n_{1} \kappa_{2}, n_{2} \kappa_{1}, \delta_{1}+\delta_{2}\right\} .
$$

Theorem 2. For every two nontrivial strongly connected digraphs $G_{1}$ and $G_{2}$,

$$
\lambda\left(G_{1} \times G_{2}\right)=\min \left\{n_{1} \lambda_{2}, n_{2} \lambda_{1}, \delta_{1}^{+}+\delta_{2}^{+}, \delta_{1}^{-}+\delta_{2}^{-}\right\} .
$$

Proof. To prove the equality, we only need to prove that

$$
\lambda\left(G_{1} \times G_{2}\right) \geq \min \left\{\lambda_{1} n_{2}, \lambda_{2} n_{1}, \delta_{1}^{+}+\delta_{2}^{+}, \delta_{1}^{-}+\delta_{2}^{-}\right\}
$$

since the reverse inequality clearly holds. Let $G=G_{1} \times G_{2}$ and $B$ be a minimum edge-separating set of $G$.
Suppose that $C_{1} \neq V_{1}$ and $C_{2} \neq V_{2}$ for each strongly connected component $C$ of $G-B$. Then

$$
\begin{array}{ll}
\left|B_{x}\right| \geq 1 & \text { for each } x \in V_{1} \text { and } \\
\left|B^{y}\right| \geq 1 & \text { for each } y \in V_{2} .
\end{array}
$$

Thus,

$$
|B|=\sum_{x \in V_{1}}\left|B_{x}\right|+\sum_{y \in V_{2}}\left|B^{y}\right| \geq n_{1}+n_{2} \geq \delta_{1}^{+}+\delta_{2}^{+}+2 .
$$

Now, suppose there exists such a strongly connected component $C$ of $G-B$ where either $C_{1}=V_{1}$ or $C_{2}=V_{2}$. By symmetry, we can without loss of generality assume $C_{1}=V_{1}$. Let $C^{\prime}(\neq C)$ be a strongly connected component without out-neighbors in $G-B$. Note that

$$
\left|B_{x}\right| \geq \lambda_{2} \quad \text { for } x \in C_{1}^{\prime} .
$$

If $C_{1}^{\prime}=V_{1}$, then from (7) we have

$$
|B| \geq \sum_{x \in C_{1}^{\prime}=V_{1}}\left|B_{x}\right| \geq n_{1} \lambda_{2} .
$$

If $C_{1}^{\prime} \neq V_{1}$, then by Lemma 1 there exists an edge $\left(x, x^{\prime}\right) \in$ $E_{G_{1}}^{+}\left(C_{1}^{\prime}\right)$ such that $\left|B_{x}\right|+\left|B_{x x^{\prime}}\right| \geq \delta_{2}^{+}+1$. And we can also see that

$$
\left|B_{e}\right| \geq 1 \quad \text { for each } e \in E_{G_{1}}^{+}\left(C_{1}^{\prime}\right) .
$$

It follows from (1), (8), and Lemma 2 that

$$
\begin{aligned}
|B| & \geq\left|B_{x}\right|+\left|B_{x x^{\prime}}\right|+\sum_{z \in C_{1}^{\prime} \backslash\{x\}}\left|B_{z}\right|+\sum_{e \in E_{G_{1}}^{+}\left(C_{1}^{\prime}\right) \backslash\left\{x x^{\prime}\right\}}\left|B_{e}\right| \\
& \geq \delta_{2}^{+}+1+\left|C_{1}^{\prime}\right|-1+\left|E_{G_{1}}^{+}\left(C_{1}^{\prime}\right)\right|-1 \\
& =\delta_{2}^{+}+\left(\left|C_{1}^{\prime}\right|+\left|E_{G_{1}}^{+}\left(C_{1}^{\prime}\right)\right|\right)-1 \\
& \geq \delta_{2}^{+}+\left(\delta_{1}^{+}+1\right)-1 \\
& =\delta_{1}^{+}+\delta_{2}^{+} .
\end{aligned}
$$

This completes the proof.

Corollary 2. ([12]) For every two nontrivial connected undirected graphs $G_{1}$ and $G_{2}$,

$$
\lambda\left(G_{1} \times G_{2}\right)=\min \left\{n_{1} \lambda_{2}, n_{2} \lambda_{1}, \delta_{1}+\delta_{2}\right\} .
$$

\section{REMARKS}

It is well known that the Cartesian product $G_{1} \times G_{2}$ of two graphs $G_{1}$ and $G_{2}$ gives an important method for designing large-scale interconnection networks. For example, the very popular, versatile, and efficient hypercube $Q_{n}$ can be expressed as $Q_{n}=\underbrace{K_{2} \times K_{2} \times \cdots \times K_{2}}_{n}$, where $K_{2}$ is a complete graph of order two. In this note, we completely determine the connectivity and the edge-connectivity of $G_{1} \times G_{2}$ for two strongly connected digraphs $G_{1}$ and 
$G_{2}$, which are equal to $\min \left\{n_{1} \kappa_{2}, n_{2} \kappa_{1}, \delta_{1}^{+}+\delta_{2}^{+}, \delta_{1}^{-}+\delta_{2}^{-}\right\}$ and $\min \left\{n_{1} \lambda_{2}, n_{2} \lambda_{1}, \delta_{1}^{+}+\delta_{2}^{+}, \delta_{1}^{-}+\delta_{2}^{-}\right\}$, respectively; these generalize two corresponding results for undirected graphs, namely Corollary 1 and Corollary 2 . As another application, we immediately obtain $\kappa\left(Q_{n}\right)=\lambda\left(Q_{n}\right)=n$.

We note that Chartrand and Harary [3] introduced permutation graphs. For a graph $G$ and a permutation $\pi$ of $V(G)$, the permutation graph $G^{\pi}$ is defined by taking two disjoint copies of $G$ and adding a matching joining each vertex $v$ in the first copy to $\pi(v)$ in the second copy. The connectivity and edge-connectivity of $G^{\pi}$ were studied in $[2,5,7]$. It is clear that permutation graphs cannot contain the Cartesian product graphs. However, if we take $\pi$ in $G^{\pi}$ as the identity permutation, then the permutation graph $G^{\pi}=G \times K_{2}$.

We also note that Balbuena et al. [1] defined the product graph $G_{1} \times G_{2}$ of two undirected graphs $G_{1}$ and $G_{2}$, which is a generalization of both the Cartesian product graphs and the permutation graphs. Thus, it is interesting to determine the connectivity and edge-connectivity of $G_{1} \times G_{2}$.

\section{REFERENCES}

[1] C. Balbuena, P. García-Vázquez, and X. Marcote, Reliability of interconnection networks modeled by a product of graphs, Networks 48 (2006), 114-120.

[2] C. Balbuena, X. Marcote, and P. García-Vázquez, On restricted connectivities of permutation graphs, Networks 45 (2005), 113-118.
[3] G. Chartrand and F. Harary, Planar permutation graphs, Ann Inst H Poincaré Sec B 3 (1967), 433-438.

[4] W.-S. Chiue and B.-S. Shieh, On connectivity of the Cartesian product of two graphs, Appl Math Comput 102 (1999), 129-137.

[5] W. Goddard, M.E. Raines, and P.J. Slater, Distance and connectivity measures in permutation graphs, Discrete Math 271 (2003), 61-70.

[6] M. Lu, G.-L. Chen, and J.-M. Xu, On super edge-connectivity of Cartesian product graphs, Networks 49 (2007), 135157.

[7] B. Piazza, Edge-connectivity of permutation graphs, Congr Numer 65 (1988), 7-16.

[8] G. Sabidussi, Graphs with given group and given graph theoretical properties, Can J Math 9 (1957), 515-525.

[9] J.-M. Xu, Connectivity of Cartesian product digraphs and fault-tolerant routings of generalized hypercubes, Appl Math J Chin Univ B 13 (1998), 179-187.

[10] J.-M. Xu, Toplogical structure and analysis of interconnection networks, Kluwer Academic Publishers, Dordrecht, 2001.

[11] J.-M. Xu, Theory and application of graphs, Kluwer Academic Publishers, Dordrecht, 2003.

[12] J.-M. Xu and C. Yang, Connectivity of Cartesian product graphs, Discrete Math 306 (2006), 159-165.

[13] J.-M. Xu and C. Yang, Connectivity and super-connectivity of Cartesian product graphs, Ars Combinatoria, accepted. 